\title{
BESP: An Integrated Artificial Intelligence-Based Platform for Building and Environment Simulation
}

\author{
Walter Mazuroski ${ }^{1}$, Ricardo C.L.F de Oliveira ${ }^{2}$, Nathan Mendes ${ }^{1}$ \\ ${ }^{1}$ PUCPR/PPGEM/LST, Pontifical Catholic University of Parana, Curitiba, PR, Brazil \\ ${ }^{2}$ School of Electrical and Computer Engineering, University of Campinas, Campinas, SP, Brazil
}

\begin{abstract}
A new Building and Environment Simulation Platform (BESP) has been conceived to be an advanced multidimensional simulation platform for fast and accurate assessment of energy and hygrothermal performance of building elements, buildings and building communities by means of an intelligent co-simulation approach among building energy simulation tools and specialized software. The simulation platform has also been idealized to be a user-friendly tool that enables to bring Computational Fluid Dynamics (CFD) to different building energy simulation programs such as EnergyPlus, Domus and Modelica among others. The platform is open for contributions from researchers or students who wish to help on the development of the contents of the toolbox by adding, for instance, new FMUs (Function Mock-Up Units). This paper presents the platform structure and backbone. The concept and one example of co-simulation is provided, illustrating some of the potential of the new simulation platform.
\end{abstract}

\section{Introduction}

A great number of building performance simulation (BPS) tools are currently available. Most of tools have been developed for comprehensive unsteady energy analyses (BLAST, BSim, Domus, EnergyPlus, ESP-r and TRNSYS), some of them since early seventies due to the energy crises. Since early nineties, more sophisticated wall models have emerged to consider not only heat transport but also moisture adsorption/desorption effects. Despite this great number of tools and models that have appeared during the last 50 years, building energy simulation tools still considerably simplify the physics, especially regarding three-dimensional convective and diffusive transport phenomena (heat, air and moisture). In addition, components, equipment and systems, are commonly represented by steady-state, lumped or empirical models. All those simplifications may significantly increase the gap between reality and simulation. Mendes et al. (2016) presented a brief history on heat and moisture modelling and a view on the trends for building simulation tools (see Figure 1). According to the authors, in the upcoming years, with the interest of smart cities and urban physics, building simulation moves to the city scale, which greatly increases the challenges.

Moreover, simulation tools must be prepared to face the challenges related to the fourth industrial revolution and to be easily expanded to include advanced building elements and systems that might arise rapidly in the market. Furthermore, they should be also prepared to exchange models and be open to communicate to thirdpart tools.

Therefore, an innovative and modular conception is needed for the upcoming generation of building energy simulation tools. In this new conception, users can work individually on model development and the results can be easily explored by all building simulation tools.

Some modular tools emerged in the past such as ASTECCA (Mendes et al. 2003), the International Building Physics Toolbox (IBPT), SIMBAD (SIMBAD, 2005) and CARNOT (Wemhöner et al. 2000) as specialized tools in the building services area, while others such as HamLab (Schijndel and Hensen, 2005) focused on building physics. Those tools were developed to run over Matlab/ Simulink platform to be more flexible, modular, transparent and an easy-to-use developing environment and to the availability of libraries, including the state-of-the-art ordinary differential equation (ODE) solvers.

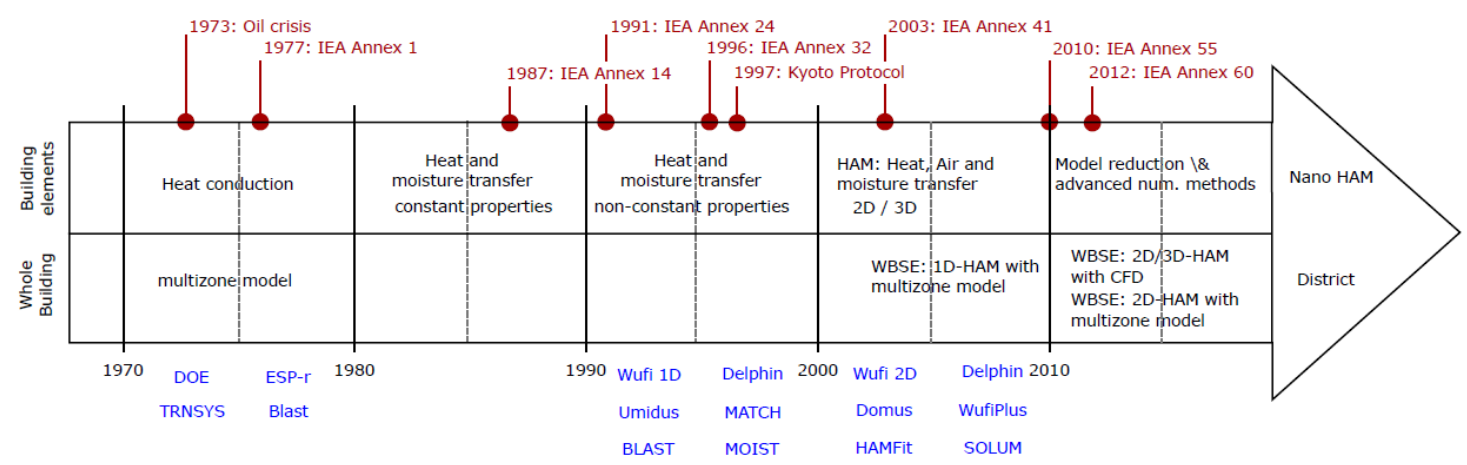

Figure 1: A brief history on heat and moisture modelling for building simulation tools (Mendes et al. 2016). 
However, the computer run time to simulate wholebuildings is an important constraint associated to the use of those Matlab/ Simulink based tools, even by using only lumped approaches in the air domain, i.e., based on a simple integral formulation of conservation equations that assume air as perfect mixed within the thermal zone space.

In this way, a new simulation platform is proposed as a toolbox with a methodology to bring advanced physics into the building simulation, by integrating different simulation tools and specialized software such as the packages for computational fluid dynamics. Thanks to the use of the methodology of intelligent co-simulation presented in Mazuroski et al. (2018) and Berger et al. (2018), the computer run time can be drastically reduced even for 3D whole-building simulation.

In addition, this simulation platform enables to make use of the best features of different BPS tools. For instance, the pixel counting available in Domus (Rocha, Oliveira and Mendes 2017; Rocha, et al. 2019) allows accurate and fast calculation of sunlit areas on all external and internal building surfaces regardless the complexity of shading elements. On the other hand, co-simulation of Modelica libraries enables to use detailed information for considering control of HVAC systems among other features. Modelica libraries that are part of the Modelica IBPSA Library are developed for design and operation through and can also be integrated to the platform by means of a FMI (Functional Mockup Interface).

In the article, we first present a brief discussion on the simplification of transport phenomena in buildings. Then, we describe the methods, including the BESP structure, co-simulation method, the intelligent co-simulation feature and some examples to describe the building simulation platform.

\section{Transport Phenomena in BPS tools}

Contemporary BPS (Building Performance Simulation) tools are typically used to predict performance of buildings for long periods such as a whole year. As a result, the representations of certain physical phenomena are simplified, or even neglected, for instance, the 1-D treatment of heat conduction through the building envelope and foundation. These simplifications certainly reduce the precision to represent complex physical phenomena associated to non-uniform conditions of convective and radiative boundaries, thermal bridges and complex geometries. Such issues have been studied in a few publications, for example, in Rodler, Virgone and Roux (2016) the effect of a more complex 3-D thermal analysis in a low energy building is described. The 3-D thermal envelope model is more accurate to describe the air, the surface temperature field and the heating load requirement. Comparisons with a 1-D thermal model and on-site experiments presented important discrepancies for the assessment of surface temperatures and heating power when using a simplified model. Moreover, a 3-D model is more adapted than 1-D models when the objective is to evaluate the behavior of surfaces composed of different materials with different thermal masses (Rodler, 2014).

The evaluation of heat losses through thermal bridges and their effect on the overall building performance represent a difficulty frequently faced in building simulation (Déqué, Ollivier and Roux 2001). The dynamic effect of thermal bridges on the building energy performance is evaluated in Ge and Baba (2015) using WUFI Plus software, modeling the thermal bridges using a 3-D dynamic method and the equivalent U-value method. Simulation results show that the annual heating load is underestimated by $12.5-14.8 \%$ using the equivalent Uvalue method compared to the $3-\mathrm{D}$ purely diffusive transient method.

CFD is currently an option to perform more complex predictions in buildings physics, by including detailed 3D modelling of heat and air transport. According to Wurtz, Nataf and Winkelmann (1999), the major difficulty with CFD - especially in three dimensions - is that the calculations are very slow and require large amounts of memory. An intermediate approach that allows to determine the airflow pattern inside a room without the computational investment of CFD is the "zonal method" which is adapted to a building domain characterized by great volumes. However, this method has shown a reasonable accuracy only for simple rectangular geometries.

To bring the CFD approach into the building simulation field, some authors have used co-simulation techniques. For instance, Mazuroski et al. (2017) included multidimensional effects, natural convection airflow, non-uniform convective heat transfer coefficients by means of co-simulation. It is shown how the zone temperature prediction can be highly improved in terms of accuracy with the co-simulation between a BPS and a Computational Fluid Dynamics (CFD) tool. The results of a co-simulation of a triangular-prism-shaped attic model showed that a good improvement in terms of accuracy can be achieved, although the technique presents as a drawback a high computational cost.

In Berger et al. (2016), a co-simulation was performed between a building simulation program and an in-house reduced order model for simulating 2-D heat and moisture transfer through a porous wall. Among other examples arising from the co-simulation in building physics, one can mention the one presented in Zuo (2016) that proposes a co-simulation to deal with the interactions among room airflow, heat, ventilation and air conditioning (HVAC) systems and building envelope, within the outlooks of management of systems. In Hensen et al. (2004), the reduction on emission of greenhouse gases and the substantial improvements on health, comfort and productivity based on co-simulation between tools is mentioned.

Therefore, innovative and fast simulation/co-simulation methods are needed to enable detailed and accurate simulation of buildings, including 3-D heat/moisture transfer and airflow simulation, considering non- 
homogeneous distribution of radiative boundary conditions. This need has inspired the development of BESP to bring complex physics in building performance assessment via the intelligent co-simulation approach presented in Mazuroski et al. (2018).

\section{Methods}

\section{Co-simulation}

Co-simulation can be technically defined as a type of simulation where at least two simulation tools - each one offering different numerical solutions to a physical or mathematical problem - jointly solve differentialalgebraic systems of equations, exchanging data while the coupling is active (IEA Annex 60 2012, Nouidui et al. 2014, Mazuroski et al. 2017).

Improvements in building analysis could be achieved with the help of a BPS tool when buildings and their systems are treated as a complete optimized entity. It is a fact that the development team of a BPS tool needs to have an indepth knowledge of the software architecture, programming language, hardware support, modelling approaches and strategies, and due to the rapid innovations in building and system technologies, to keep a BPS tool continuously updated is a hard task. In this context, co-simulation can be an interesting alternative (Trcka, Hensen and Wetter 2009).

There are some co-simulation techniques and combination of methods such as the one-to-one approach and the FMI standard. In the one-to-one approach, a specific protocol regulates the exchange of data during run time. It can be considered as the most flexible approach and can support any kind of numerical solution method (Wetter and Treeck 2017). For instance, a one-toone coupling was adopted to connect Domus to the commercial CFD tool ANSYS-CFX. The type of coupling used was the Ping-Pong method (Hensen 1999), creating a weak coupling between the tools. The one-toone approach requires a specific implementation in each of the tools to be coupled. Such demands require implementation efforts over time to keep the compatibility between the tools. To overcome these limitations, an open-source standard for coupled simulation, known as FMI Standard, was proposed by Modelisar project in 2008. The use of the FMI standard provides greater scope for co-simulation between tools that adopt the same standards such as EnergyPlus, Modelica Models and many others available at https://fmi-standard.org.

A review of co-simulation examples can be found in some works in the frame of the IEA Annex 60 (Wetter and Treeck 2017) as well as Wetter (2011) and Berger et al. (2018).

\section{BESP: Building Environment Simulation Platform}

BESP has been developed to be an advanced multidimensional simulation platform for fast and accurate assessment of energy and hygrothermal performance of building elements (glazing/opaque components, innovative systems etc.), buildings (smart systems etc.) and building communities (smart grid, smart city) by means of intelligent co-simulation (Mazuroski, et al. 2018; Mazuroski, et al. 2017) among building energy simulation tools (EnergyPlus, Domus, Modelica) and specialized software. The simulation platform has also been conceived to be a user-friendly tool that enables to bring Computational Fluid Dynamics (CFD) to different building energy simulation programs such as EnergyPlus, Domus and Modelica among others. Any researcher and student can help on the development of the contents of the toolbox by adding new FMUs (Function Mock-Up Units). BESP has been built in $\mathrm{C}++$ Builder and designed to integrate different tools by means of FMI 1.0 interface for model exchange and python scrips to provide cosimulation with a CFD tool. The platform program runs in the Windows operating systems. The user interface consists of a series of windows in which the user can enable the relevant input data (since a building element to a city), the BPS software to be run, the Modelica or python model or CFD package to be co-simulated with and the post-processors files to review the results.

The use of BESP may enable to consider the best features of different BPS tools and combine to CFD packages for modeling improvement of processes such as ventilation, interzone airflow, pollutants transport, moisture migration and microclimate and heat island analyses. In addition, BESP enables the co-simulation of building districts, considering the convective phenomena around buildings and short- and long-wave radiation. The use of CFD to simulate a room, a building or a district becomes possible thanks to the intelligent co-simulation technique, which allows to perform extremely timeconsuming simulations over a reasonable period.

\section{BESP structure}

Figure 2 illustrates the BESP structure composed by cosimulation using FMI standard and a python script-based co-simulation, a set of plug-ins to provide services such as energy efficiency and thermal analysis according to regulations and certifications and the Intelligent cosimulation feature to make possible the use of complex physics as the one available in CFD tools for fast and high-precision analysis.

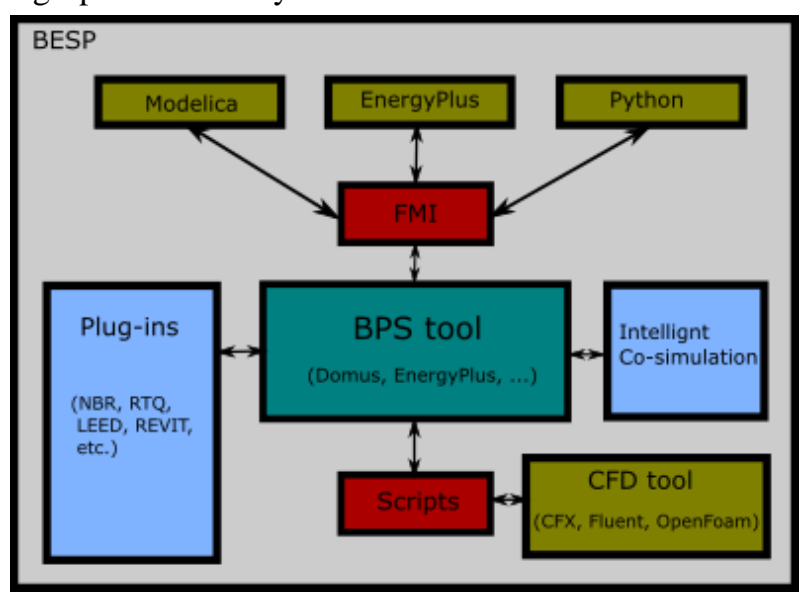

Figure 2: BESP structure. 


\section{BESP co-simulation}

The one-to-one and FMI approaches are implemented in BESP and enable its coupling with other models and tools. For the co-simulation between a BPS tool and a CFD tool, the one-to-one coupling based on python scripts is used. In the case of co-simulation for coupling of models, the FMI 1.0 standard was chosen, allowing to use models created in Modelica and models developed directly in python language. As an example, Figure 3 shows the setup interface of a python model using FMI for co-simulation, where it is defined the boundary conditions that are exchanged between the BPS tool and the coupled model. Such interface was devised to be userfriendly, requiring only the path to the FMU file and some associations among the variables.

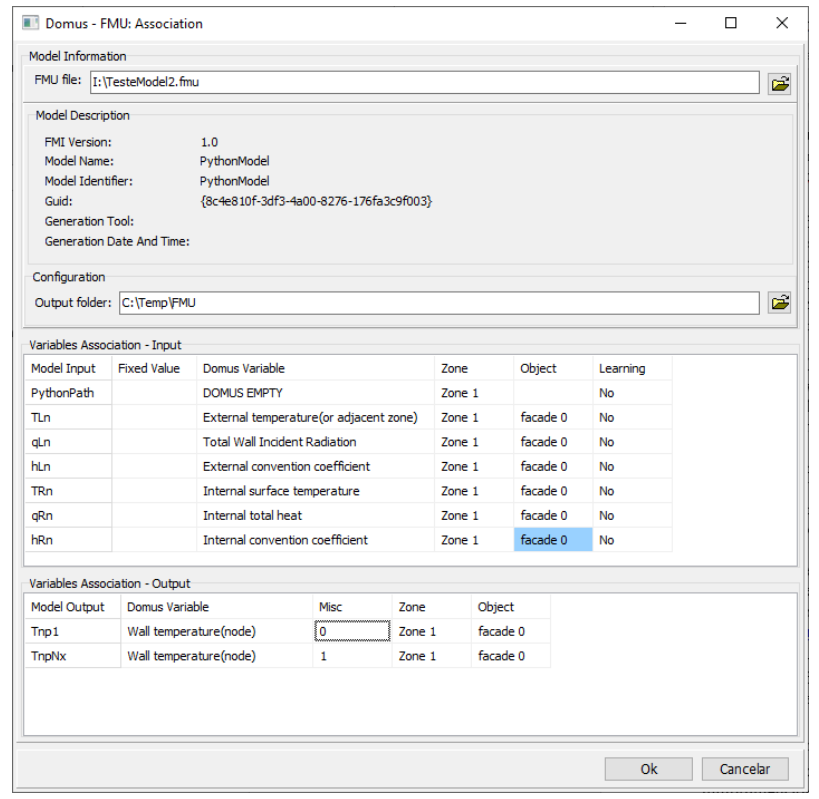

Figure 3: Model setup interface.

As a distinct feature of the tool, a C++ DLL was developed to provide the interface between the FMI standard and a python-developed model. This DLL must be included inside the FMU file, as well as some small .py configuration files edited by the user. This FMU enables to execute Python models that can act in the form of cosimulation and directly influence the behavior of the building. Some examples of use - but not limited to - are wall and window models.

\section{Intelligent co-simulation approach}

The intelligent co-simulation approach was first proposed in Mazuroski et al. (2018), and improved versions were detailed in Berger et al. (2018), considering Domus, as a building performance simulation tool, and CFX as a CFD specialized software. As described in Mazuroski et al. (2018) there is a significant computational effort involved in the co-simulation approach. Therefore, the idea of intelligent co-simulation is to have a machine learning model that can stop the communication between the two programs and replace one of them. The intelligent cosimulation is composed of two phases: i) the training phase and ii) the prediction phase. Thus, as soon the prediction model is trained, CFX is disconnected from the co-simulation and Domus performs the rest of the simulation using the prediction model, being much faster to simulate with a low impact on the accuracy of the results.

An important improvement in the intelligent cosimulation technique was presented in Berger et al. (2018), based on the definition of the training climate to build an accurate prediction model. In its original version, the intelligent co-simulation used the first days of the simulation period to perform the training. In the improved version presented in in Berger et al. (2018), a new strategy was used to specify periods of training, using a set with the hottest and coldest days of the year.

An implementation of the intelligent co-simulation approach is available in the BESP and provide capability to speed-up a complex simulation by means of machine learning technology.

\section{Plug-ins}

Specialized plug-ins are planed to be available into the BESP funcionalities to provide useful resouces for the building simulation user community. Plug-ins to perform building rating based on certifications such as LEED (Leadership in Energy and Environmental Design) from United States Green Building Council and Brazilian equivalent standards, NBR and RTQ, are also under development.

\section{Case Study}

The detailed building model presented as case study in this work comprises a two-storey building as illustrated in Figure 4 . With approximately $723 \mathrm{~m}^{3}$ total volume, the building is composed by four 3-mm glazing windows, 1 wood door, a 20-mm tiled roof, 100-mm concrete walls, a $2 \mathrm{~m} \times 4 \mathrm{~m}$ opening between the two storeys with a 13-step ladder $(2 \mathrm{~m} \times 0.1 \mathrm{~m} \times 0.3 \mathrm{~m})$, as depicted in Figures 5 and 6.

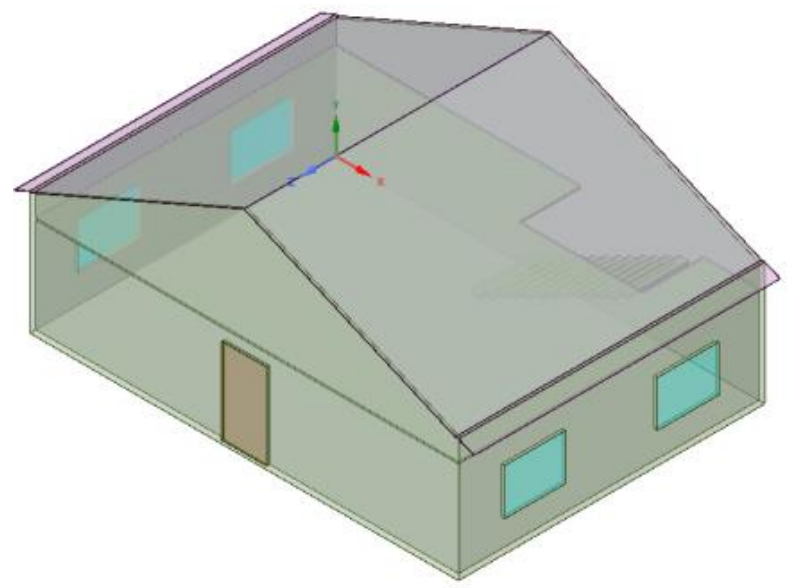

Figure 4: Isometric view of the case study in the SpaceClaim graphical interface. 


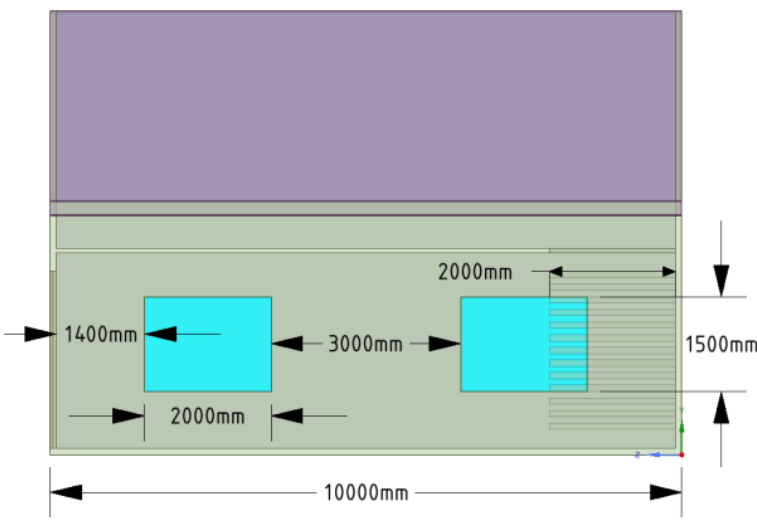

Figure 5: Details of case study building - west view.

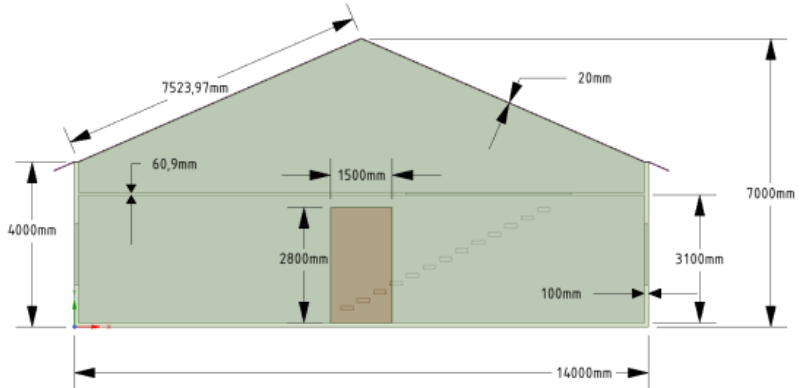

Figure 6: Details of case study building - south view.

Domus has been chosen as the BPS tool due to the full understanding and access to the source code by the authors. Moreover, currently, only Domus has the pixel counting technique for sunlit calculation integrated to the calculation engine. The chosen CFD tool was ANSYSCFD.

Previously described in Mazuroski et al. (2018), Figure 7 illustrates the communication between Domus and CFX, where Domus is the master of the simulation. At the initial stage, the building envelope thermophysical properties ( $\rho$, $c, \lambda)$ and the boundary conditions are informed to CFX. The latter computes a steady-state simulation step as initial conditions.

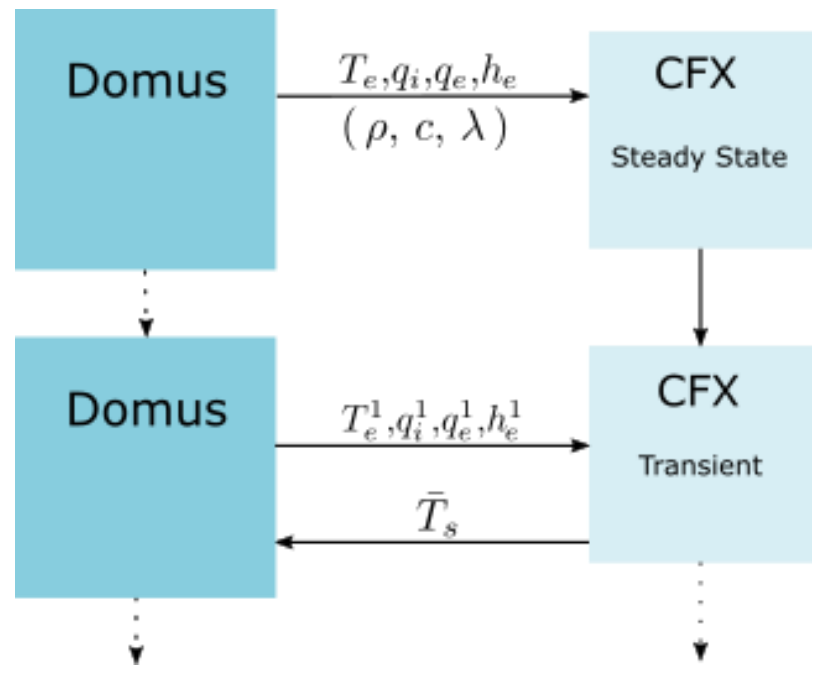

Figure 7: Co-simulation scheme between Domus and CFX. Source: Mazuroski et al. (2018).
The common information used in the entire building is the external weather temperature $\left(T_{e}\right)$, the outside convective heat transfer coefficients $\left(h_{e}\right)$ and the external and internal heat fluxes $\left(q_{e}, q_{i}\right)$. An additional information presented in this work for boundary conditions is the sunlit for all the internal and external surfaces. At each time step, Domus provides the outside and inside boundary conditions $\left(T_{e}, q_{e}, q_{i}, h_{e}\right)$ for each surface to CFX.

The CFX simulates the whole 3-D building model, considering the natural convection in the air domain and the 3-D diffusive heat transfer in all solid parts. After a successful run, CFX returns to Domus the desired output variable, for example the spatially averaged surface temperature $\left(\bar{T}_{s}\right)$ of all building internal surfaces that have been considered in the Domus-CFX coupling.

The CFD coupled model receives non-uniform boundary conditions during the run time period of simulation. This coupling requires the pre-definition of a template of the target geometry, as well as the setup of the mesh by the user. The information transmitted to the CFD tool, such as air temperature, incident radiation flux and convective heat transfer coefficients, are from the previous time-step simulation.

For the CFD, an adaptive mesh is generated for the building geometry and, based on a grid convergence index (GCI) calculation (Roache, 1994), with limitations imposed by the available computational capacity, a mesh of 420 thousand nodes presents good mesh independent results, since a greater refinement does not bring considerable difference in the results of the model. The thermal parameters of different materials used in the simulation are listed in Table 1. Simulations were performed using a computer equipped with Windows 864 bit and an Intel Core i7 4790 Processor with a four-core CPU at $3.6 \mathrm{GHz}$. The CFX used three cores for the simulation. The co-simulation time step was set to $6 \mathrm{~min}$ and the total horizon time of co-simulation was 2 weeks (14 days).

Table 1: Range of parameters used in simulation.

\begin{tabular}{|c|c|c|c|c|}
\hline \multirow{2}{*}{ Parameter } & \multicolumn{4}{|c|}{ Material } \\
\cline { 2 - 5 } & Air & $\begin{array}{c}\text { Roof } \\
\text { Tile }\end{array}$ & $\begin{array}{c}\text { Concrete } \\
\text { Slab }\end{array}$ & Wood \\
\hline Density $\left(\mathrm{kgm}^{3}\right)$ & 1.200 & 2000 & 2200 & 720 \\
\hline $\begin{array}{c}\text { Specific heat } \\
\left(\mathrm{Jkg}^{1} \mathrm{~K}^{1}\right)\end{array}$ & 1007 & 920 & 1000 & 1255 \\
\hline $\begin{array}{c}\text { Thermal } \\
\text { conductivity } \\
\left(\mathrm{Wm}^{1} \mathrm{~K}^{1}\right)\end{array}$ & 0.024 & 1.05 & 1.75 & 0.16 \\
\hline
\end{tabular}

\section{Boundary and Initial Conditions}

By means of the co-simulation, Domus provides all boundary conditions to CFX. Providing the weather temperature, external convective heat transfer coefficient, direct, diffuse and reflected radiation fluxes, and the sunlit contour on each internal and external surface of the building. 
The initial temperature is $T_{i}=20^{\circ} \mathrm{C}$ The convective heat transfer coefficients are set to $h_{e}=23 \mathrm{~W} /\left(\mathrm{m}^{2} . K\right)$. The outside conditions represent a temperate climate of Curitiba, Brazil. The inside temperature and relative humidity vary freely. Due to the extremely high computational cost, a two-week simulation period was chosen. One week including the winter solstice in the southern hemisphere, starting at June 21, and the other one on the summer solstice, starting at December 21. The objective is to closely analyses the results on days 21 and 22 to verify the thermal-physical behavior. The complete result of day 21 to 27 are used to evaluate the prediction model accuracy.

The boundary conditions for the 3-D building model consist of 38 input variables, provided by Domus.

\section{Results}

In order to explore the air temperature and velocity behaviors within the building space, major advantage of the physical simulation in three dimensions, virtual nomass sensors are detailed in this section.

To better illustrate the potential of the present approach, this paper presents results from the winter period in Figures 8, showing the airflow computed by the CFD tool and in Figure 9 the temperature contour plot and velocity vector field in a vertical plane. In general, the airflow calculated by means of CFD codes may improve the accuracy of BPS tools, but at a prohibitive simulation cost. As one can observe, the airflow varies significantly along the day and a constant value, which is normally used by BPS tools, may significantly affect the results. Indeed, the magnitude of this airflow could be greatly magnified if a heater or a fan had been considered and traditional lumped-based simulation tools would not be able to provide accurate/reasonable results.

The entire 14-day simulation period took approximately 1200 hours of computation (50 days). An average time of 18 minutes per time step is consumed by CFX.

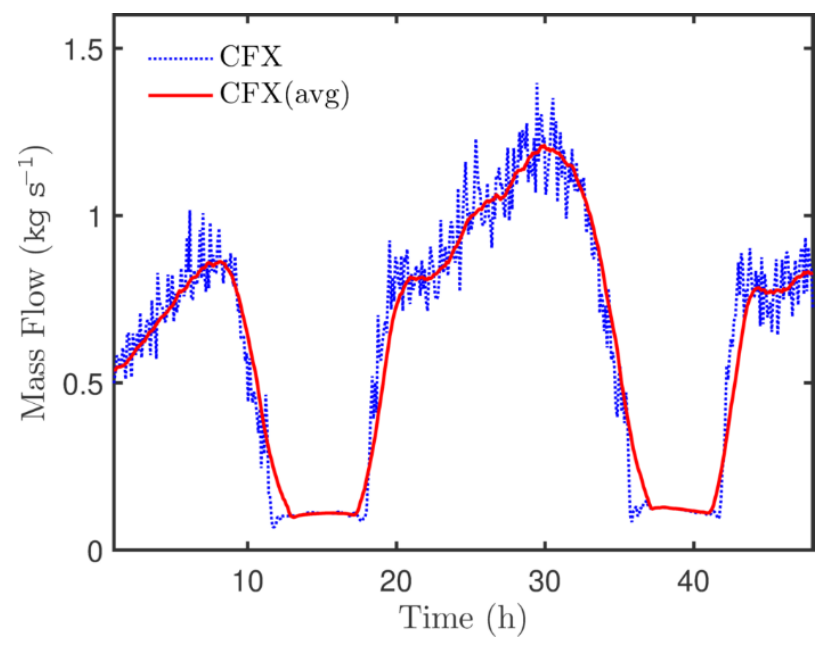

Figure 8: Mass airflow at the opening, on 21st and 22nd of June.

\section{Intelligent Co-Simulation}

The RNN (Recurrent Neural Network) used in the intelligent co-simulation approach managed to predict satisfactorily the airflow, (see Figure 10), to be used very rapidly in annual simulations, even varying the external boundary conditions, i.e., simulating the same building for other climates. The models built to predict the mass airflow were trained by using the representative climate.

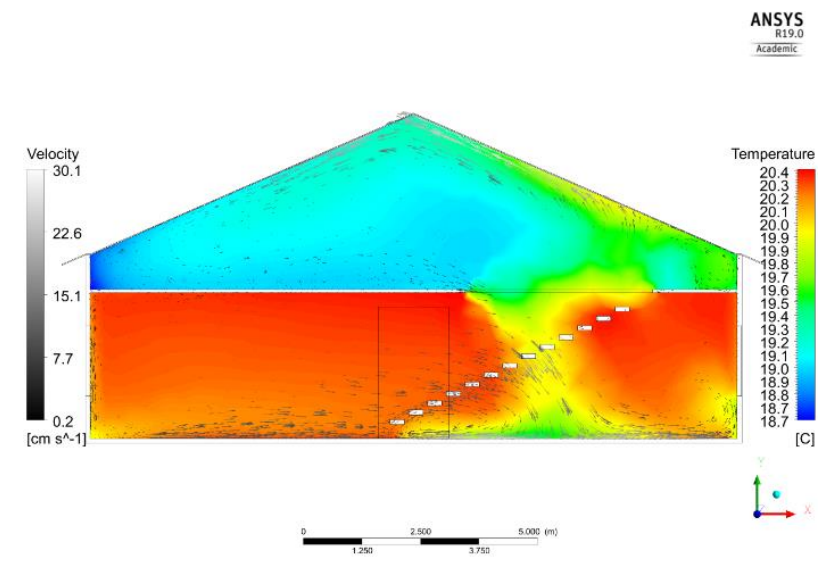

Figure 9: Contour plot of the air local range temperature, overlaid by the velocity vector field, calculated at a vertical plane at $1 \mathrm{~m}$ from the west wall.

The computational time for the 14-day simulation using the RNNs model is of the order of seconds, i.e., much faster than the detailed Domus-CFX model. Once trained, the prediction model can be stored for further usage.

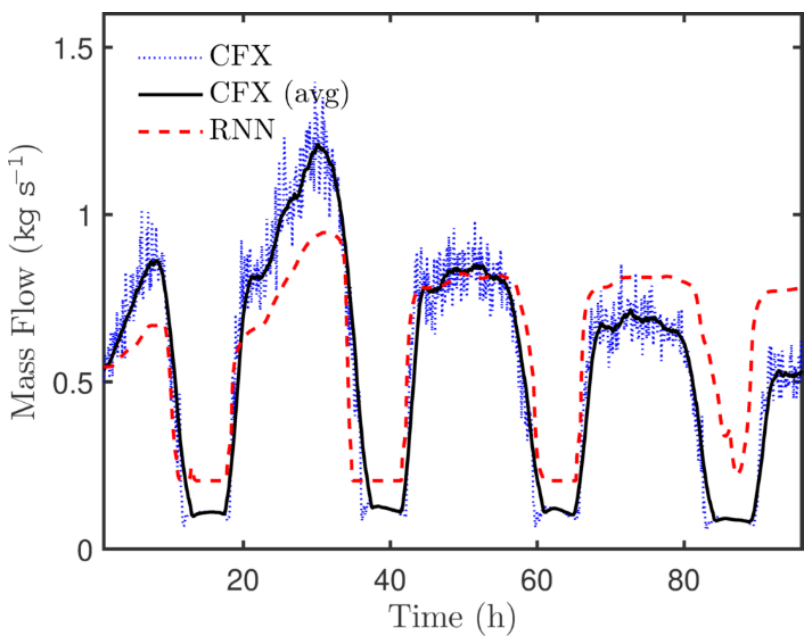

Figure 10: Mass airflow at the opening, on 21st and 22nd of June.

It is important to note that because of the rapid response present in airflow behavior, the prediction becomes more challenging than it would be for more compliant systems such as air or solids temperature. A 14-day training period with a 6-minute time step generates a relatively small amount of data for the training set. A larger set of training would certainly bring a considerable improvement in prediction. One of the great advantages of the method is 
to use the principles of machine learning and accumulate training to generate an increasingly powerful database in predicting the physical behavior of the building.

The training phase generated 8 RNNs, one for each sensor and type of training. All with only one hidden layer and number of nodes ranging between 1 and 9, the networks to predict the air velocity tended to form networks with less nodes in the hidden layer.

\section{Conclusion}

This paper described the Building and Environment Simulation Platform (BESP), conceived to bring advanced physics to building performance assessment.

The structure and the methods are first described. Then, a one-to-one co-simulation approach is presented. Preliminarily, within the BESP structure, Domus-CFX was used to calculate the transient airflow through a ladder way of a two-storey house.

BESP applied the RNN technique in the frame of the intelligent co-simulation approach, which managed to predict the transient mass flow, to be used very rapidly in annual simulations, even varying the external boundary conditions, i.e., simulating the same building for other climates, as shown in previous intelligent co-simulation publications (Mazuroski et al. 2018). The models built to predict the mass airflow were trained by using a representative climate so that it can be applied in cities with similar climate data with no need to co-simulate again with the specialized CFD tool. Moreover, unlike simulation tools based on integral approaches for the air domain, BESP can also provide results in terms of temperature, air pressure and air velocity as a function of time and position in the building, with no additional cost after the accomplishment of the prediction phase.

Therefore, it has been seen the advantage of combining different tools (BPS and CFD) with an innovative cosimulation method based on machine learning. In future versions, the platform will be also prepared to co-simulate with FMUs dedicated to photovoltaic systems and HVAC systems. The next step, within the BESP development framework, is to combine EnergyPlus and a CFD tool CFX, Fluent or OpenFoam - to simulate in detail a complex building by means of the intelligent cosimulation approach.

\section{References}

Berger J., Mazuroski W., Guernouti S., Mendes N. and Woloszyn M. (2016). 2D whole-building hygrothermal simulation analysis based on a PGD reduced order model. Energy and Buildings 112:4961, January 2016.

Berger, J., Mazuroski, W., Oliveira, R. C. L. F. and Mendes, N. (2018). Intelligent co-simulation: neural network vs. proper orthogonal decomposition applied to a 2D diffusive problem. Journal of Building Performance Simulation 11(5), 568-587.

Déqué F., Ollivier F. and Roux J.J. (2001). Effect of $2 d$ modelling of thermal bridges on the energy performance of buildings: Numerical application on the matisse apartment. Energy and Buildings 33(6):583 - 587, 2001.

FMI-Standard. (2017). FMI-Standard Functional Mockup Interface. https://www.fmi-standard.org, 2017. Accessed: 2017-07-03.

Ge H. and Baba F. (2015). Dynamic effect of thermal bridges on the energy performance of a low-rise residential building. Energy and Buildings, 105:106$118,2015$.

Hensen J. (1999). A comparison of coupled and decoupled solutions for temperature and air flow in building. Technical report, University of Strathclyde, Glasgow, UK, July 1999.

Hensen J., Djunaedy E., Radošević M. and Yahiaoui A. (2004). Building performance simulation for better design: some issues and solutions. In Proceedings of the 21st Conference on Passive and Low Energy Architecture (PLEA), pages 1185-1190, Eindhoven, Netherlands, September 2004.

Mazuroski, W., Berger, J., Oliveira, R. C. L. F. and Mendes, N. (2018). An artificial intelligence-based method to efficiently bring CFD to building simulation. Journal of Building Performance Simulation 11(5), 588-603.

Mazuroski, W., Mendes, N., Oliveira, R. C. L. F. (2017) Co-simulation to bring advanced physics to building thermal performance analysis. Proceedings of the 15th International Building Performance Simulation Association Conference. San Francsico, CA, USA, August 2017.

Mendes, N., M. Chhay, J. Berger, and D. Dutykh. (2016). Numerical Methods for Diffusion Phenomena in Building Physics: A Practical Introduction. PUCPRess. Curitiba (Brazil).

Mendes, N., Oliveira, G. H. C., Araújo, H. X. and Coelho, L. S. (2003). A Matlab-Based Simulation Tool for Building Thermal Performance Analysis. Proceedings of the 8th International Building Performance Simulation Association Conference. Eindhoven, Netherlands, August 2003.

Nouidui T., Wetter M. and Zuo W. (2014). Functional mock-up unit for co-simulation import in EnergyPlus. Journal of Building Performance Simulation, 7(3):192-202, 2014.

Trcka M., Hensen J. L. M. and Wetter M. (2009). Cosimulation of innovative integrated HVAC systems in buildings. Journal of Building Performance Simulation, 2(3):209-230, 2009.

van Schijndel, A. W. M., and J. L. M. Hensen. (2005). Integrated heat, air and moisture modeling toolkit in Matlab. Proceedings of the 5th International Building Performance Simulation Association Conference. Montreal (Canada), 15-18 August 2005.

SIMBAD. (2005). Transient Simulation Building and HVAC toolbox. CSTB, French Center for Building 
Sciences, Marne la Vallee, France (2005), http://software.cstb.fr.

Roache P. J. (1994). Perspective: A method for uniform reporting of grid refinement studies. Journal of Fluids Engineering, 116(3):405-413, September 1994.

Rocha, A. P. A., Oliveira, R. C. L. F. and Mendes, N. (2017). Experimental validation and comparison of direct solar shading calculations within building energy simulation tools: Polygon clipping and pixel counting techniques. Solar Energy (158), 462-473.

Rocha, A. P. A., Rodler A., Oliveira, R. C. L. F., Virgone, J. and Mendes, N. (2019). A pixel counting technique for sun patch assessment within building enclosures. Solar Energy (184), 173-186.

Rodler A. (2014). Modélisation dynamique tridimensionnelle avec tache solaire pour la simulation du comportement thermique d'un bâtiment basse consommation. $\mathrm{PhD}$ thesis, 2014. Lyon, INSA 2014.

Rodler A., Virgone J. and Roux J. J. (2016). Impact of sun patch and three-dimensional heat transfer descriptions on the accuracy of a building's thermal behavior prediction. Building Simulation, 9(3):269-279, Jun 2016.
Wurtz E., Nataf J.M. and Winkelmann F. (1999). Twoand three-dimensional natural and mixed convection simulation using modular zonal models in buildings. International Journal of Heat and Mass Transfer, 42(5):923 - 940, 1999.

Wemhöner, C., Hafner, B., and Schwarzer, K. (2000). Simulation of solar thermal systems with CARNOT blockset in the environment Matlab ${ }^{\circledR}$ Simulink®. Proceedings Eurosun 2000. Copenhagen, Denmark, June 2000.

Wetter M. (2011). Co-simulation of building energy and control systems with the building controls virtual test bed. Journal of Building Performance Simulation, 4(3): 185-203, 2011.

Wetter M. and Treeck C. v. (2017). IEA EBC Annex 60: New Generation Computing Tools for Building and Community Energy Systems. September 2017.

Zuo W., Wetter M., Tian W., Li D., Jin M. and Chen Q. (2016). Coupling indoor airflow, HVAC, control and building envelope heat transfer in the Modelica Buildings library. Journal of Building Performance Simulation, 9(4):366-381, 2 